\title{
EDUCACIÓN ALIMENTARIA NUTRICIONAL (EAN) COMO CONTENIDO TRANSVERSAL EN PERSPECTIVA DE POLÍTICA PÚBLICA EN INSTITUCIONES DE NIVEL INICIAL Y PRIMARIO DE LA CIUDAD DE ROSARIO, PROVINCIA DE SANTA FE
}

\author{
Victoria Bruch * \\ Universidad del Centro Educativo Latinoamericano, Argentina \\ victoriabruch@hotmail.com \\ Natalia Retamar Malizia ** \\ Universidad de Concepción del Uruguay Centro Regional Rosario, Argentina \\ retamarnatalia@hotmail.com \\ Ileana Silvia Sorgentoni ${ }^{* \star *}$ \\ Universidad Nacional de Rosario, Argentina \\ ileana.sorgentoni.85@gmail.com
}

Recibido: 10/06/2020 - Aceptado: 27/01/2021

\begin{abstract}
Resumen
Este trabajo presenta el desafío de pensar a la Educación Alimentaria Nutricional (EAN) en clave de política pública, como un derecho de acceso igualitario para niños/as. El objetivo es comprender cómo se aborda la EAN en tres instituciones de gestión pública, una de Nivel Inicial y dos de Nivel Primario (Primer Ciclo) en la ciudad de Rosario, provincia de Santa Fe. Es un estudio de caso cualitativo, de tipo descriptivo, exploratorio y de corte transversal. Los datos fueron recolectados por selección y análisis documental y a través de entrevistas etnográficas. Se analizaron y contrastaron documentos y normativas internacionales, nacionales y provinciales sobre EAN, así como los contenidos curriculares que la abordan y su implementación en las instituciones locales. Los resultados arrojaron que EAN se aborda mayormente desde enfoques pedagógicos tradicionales, con escaso margen para pensar transversalmente y que la malnutrición incide negativamente en el desempeño de las trayectorias escolares. Como reflexión final se consideró que es necesario establecer EAN de manera transversal en todas las instituciones educativas públicas y privadas mediante la creación de gabinetes, con articulación y seguimiento por parte del Estado que permitan la concreción de EAN como política pública, donde se trabaje inter y transdisciplinariamente.
\end{abstract}

Palabras clave: Educación Alimentaria Nutricional - Política Pública - Trayectorias Escolares Enfoques pedagógicos.

\footnotetext{
* Licenciada en Nutrición. Universidad del Centro Educativo Latinoamericano (UCEL). Asesora en Cámara de Diputados de la Provincia de Santa Fe.

** Licenciada en Nutrición. Universidad de Concepción del Uruguay Centro Regional Rosario. Carrera de postgrado en curso: Maestría en política y gestión de la seguridad alimentaria. Universidad Nacional de Rosario.

**k Licenciada en Gestión. (Facultad, Ciencias de la Educación y Psicopedagogía. Universidad Abierta Interamericana) Diplomada en Ciencias Políticas. (Fundación Instituto Latinoamericano de Desarrollo Social y Salud. Ministerio de Educación, Cultura, Ciencia y Tecnología) Maestranda en Gestión y asesoramiento de las organizaciones educativas. (Facultad de Humanidades y Artes. Universidad Nacional de Rosario).
} 
REVISTA DE LA ESCUELA DE CIENCIAS DE LA EDUCACIÓN, AÑO 17, NRO. 16, VOL. 1, ENERO A JUNIO DE 2021. PÁGINAS 107-117. ISSN 2362-3349 (EN LÍNEA). EDUCACIÓN ALIMENTARIA NUTRICIONAL (EAN) COMO CONTENIDO TRANSVERSAL EN PERSPECTIVA DE POLITICA PÚBLICA EN INSTITUCIONES DE NIVEL INICIAL Y PRIMARIO DE LA CIUDAD DE ROSARIO, PROVINCIA DE SANTA FE. VICTORIA BRUCH.NATALIA RETAMAR MALIZIA.ILEANA SILVIA SORGENTONI.

\title{
FOOD AND NUTRITION EDUCATION AS CROSS-SECTIONAL CONTENT FROM A PUBLIC POLICY PERSPECTIVE IN INITIAL AND PRIMARY LEVEL INSTITUTIONS IN THE CITY OF ROSARIO, PROVINCE OF SANTA FE
}

\begin{abstract}
This paper presents the challenge of thinking about Food and Nutrition Education (EAN) in terms of public policy, as an equal access right for children. The objective is to understand how EAN is approached in three public management institutions, one at the Initial Level and two at the Primary Level (First Cycle) in the city of Rosario, province of Santa Fe. It is a qualitative case study, of a descriptive, exploratory and cross-sectional type. The data was collected by selection and documentary analysis and through ethnographic interviews. International, national, and provincial documents and regulations on EAN were analyzed and contrasted, as well as the curricular content that addresses it and its implementation in local institutions. The results showed that EAN is mostly approached from traditional pedagogical approaches, with little room for cross-cutting thinking, and that malnutrition has a negative impact on the performance of school trajectories. As a final observation, it was considered necessary to establish EAN in all public and private educational institutions through the creation of cabinets, with articulation and follow-up by the State, which would allow EAN to become a public policy, where work is done inter and transdisciplinarily.
\end{abstract}

Keywords: Food and Nutrition Education - Public Policy - School Trajectories - Pedagogical Approaches.

\begin{abstract}
Introducción
Este trabajo presenta el desafío de pensar a la Educación Alimentaria Nutricional (EAN) en clave de política pública, como un derecho de acceso igualitario para todo/as los/as niños/as. Plantea una serie de reflexiones, consideraciones y propuestas, en torno a la preocupación sobre qué está pasando en tres instituciones en relación a esta temática. Cuando hablamos de alimentación, hablamos de comida; los seres humanos cuando comemos no solo buscamos satisfacer una necesidad biológica, sino que le damos un significado a ese alimento y diversos usos. Comer es un acto social. Los hábitos alimentarios que acompañarán al ser humano toda la vida se construyen socialmente (Aguirre, 2010). Resulta importante que la EAN constituya un medio para el cambio social hacia la igualdad de acceso desde un acompañamiento de políticas públicas orientadas a que todas las personas en etapa de escolarización logren el máximo desarrollo personal en sus vidas. Aquí se analizan y contrastan documentos y normativas internacionales, nacionales y provinciales que refieran a EAN, así como los contenidos curriculares que la abordan para luego profundizar en su implementación en las instituciones locales. Además, se establecen entrevistas etnográficas permitiendo saber cómo se aborda, cuándo y por qué, bajo qué enfoques pedagógicos o metodologías. El estudio se realiza en la ciudad de Rosario, provincia de Santa Fe, en tres instituciones de gestión pública en contextos vulnerables: una de Nivel Inicial y dos de Nivel Primario (Primer Ciclo). El motivo de la elección de estos niveles y escuelas radica en que los contenidos y las políticas educativas en relación a la alimentación y la nutrición se desarrollan, en mayor medida, en estos niveles educativos.
\end{abstract}

\section{Justificación y problema}

La Encuesta Mundial de Salud Escolar (EMSE) 2018 en relación al sobrepeso (SP) y la obesidad (OB) mostró que el 30,3\% de los estudiantes de 13 a 17 años presenta SP y el 7,4\% OB, siendo ambos indicadores mayores en varones que en mujeres. Se observó un aumento en ambas categorías en las tres ediciones de la EMSE. Al analizar la alimentación se observó un bajo consumo de frutas y verduras en los adolescentes en general. Resulta alarmante el hecho de que un tercio de los adolescentes toma bebidas con azúcar diariamente. El aumento del consumo de alimentos ultraprocesados 1 en cadenas de comida rápida, en chicos de 13 a 15 años, respecto de la edición anterior de la EMSE, resulta consistente con múltiples fuentes de información que muestran el empeoramiento de los patrones alimentarios en la población argentina (Ministerio de Salud y Desarrollo Social de la Nación [MSAL], 2018).

En el año 2005, Argentina realizó la primera Encuesta Nacional de Nutrición y Salud (ENNyS), arrojando los primeros datos de la situación epidemiológica en torno al estado nutricional de la población. En el período 2018-2019 se llevó adelante la segunda ENNyS. Los datos de antropometría en niños, niñas y adolescentes (NNyA) de 0 a 17 años indican un escenario epidemiológico en el que conviven situaciones de déficit y de exceso de peso respecto del estado nutricional y antropométrico de la población. Sin embargo, tanto el SP como la OB resultaron ser las formas más prevalentes de malnutrición en NNyA.

${ }^{1}$ De acuerdo al sistema NOVA de clasificación de alimentos, la OPS y la OMS definen a los productos ultraprocesados como formulaciones industriales elaboradas a partir de sustancias derivadas de los alimentos o sintetizadas de otras fuentes orgánicas. 
Sobrada evidencia científica da cuenta de que el exceso de peso en NNyA condiciona la aparición de Enfermedades Crónicas No Transmisibles (ECNT) a edades más tempranas como ser Diabetes Mellitus tipo 2, Insulina Resistencia, Dislipemias, Hipertensión Arterial, Cáncer y Cardiopatías. A su vez, los resultados de la encuesta arrojaron una prevalencia de baja talla en menores de 5 años de 7,9\%, mientras que en el grupo de 5 a 17 años la misma fue de 3,7\%. La baja talla refleja una malnutrición crónica que impide a los NNyA un crecimiento adecuado. Estas cifras son más altas en la población en situación de vulnerabilidad social para todas las edades, llegando a $11,5 \%$ en el primer quintil de ingresos en la población menor de 5 años. La prevalencia de SP y de OB es preocupante en el grupo de 5 a 17 años, donde más del $40 \%$ de los NNyA tienen exceso de peso. Este indicador no presenta diferencias estadísticamente significativas por sexo, nivel educativo, cobertura de salud ni quintiles de ingresos (MSAL, 2019b).

En el año 2019, el Programa Nacional de Salud Escolar (PROSANE), publicó un estudio de valoración antropométrica al iniciar y al finalizar el ciclo de educación primaria en Argentina realizado sobre una muestra de 10.961 niños y niñas evaluados en 2012 y 2017 . El mismo concluyó que durante el ciclo primario existen cambios en las categorías nutricionales iniciales y finales con un descenso en la frecuencia de la categoría normal y aumento de los casos de SP y OB. La tasa de SP y OB al inicio del ciclo primario es elevada y aumenta significativamente al finalizar el colegio, no sólo porque los NNyA con OB no mejoran, sino porque aquellos que inician con un peso normal, finalizan la escuela primaria con SP y OB (MSAL, 2019c).

De acuerdo a las investigaciones del Centro de Estudios sobre Nutrición Infantil (CESNI) en Argentina, los patrones alimentarios han empeorado en las últimas décadas. El consumo de frutas y verduras es muy bajo; el consumo de sodio y azúcar duplica el límite máximo recomendado; y el de bebidas azucaradas es de los más altos del mundo (Zapata, 2016). Considerando los datos epidemiológicos podemos decir que Argentina se encuentra atravesando una epidemia de obesidad y otras ECNT, producto de la malnutrición crónica de la población.

La calidad de la alimentación y los entornos en los cuales se lleva adelante el acto de comer juegan un rol fundamental en el mantenimiento de la salud física, intelectual y social del ser humano. En la primera infancia ocurre el crecimiento y desarrollo psicomotriz, y a su vez, niños y niñas comienzan a incorporarse a la mesa familiar. Durante la segunda infancia, coincidiendo con la edad escolar, comienzan a compartir conductas y aprendizajes con sus pares. Por este motivo, la EAN desde edades tempranas favorece el desarrollo de hábitos beneficiosos para la salud y, a su vez, la posibilidad de sostenerlos a lo largo de la vida (Torresani, 2010).

Según lo manifiesta la Federación Argentina de Graduados en Nutrición (FAGRAN), la escuela es una institución que puede contribuir abordando la EAN tanto en el aula, como en el patio y el comedor escolar al contribuir con entornos escolares saludables, promoviendo comportamientos alimentarios que permitan mejorar la salud de la población (FAGRAN, 2013).

A raíz de este contexto, se considera necesario indagar acerca de cómo es el abordaje de EAN en estos casos y qué evidencia existe para seguir fortaleciendo el trabajo de investigación.

\section{Objetivo general:}

Comprender cómo se aborda la EAN en tres instituciones de gestión pública de Nivel Inicial y Primario en contextos vulnerables desde 2016 hasta la actualidad en la ciudad de Rosario, provincia de Santa Fe.

\section{Objetivos específicos:}

- Indagar y contrastar antecedentes nacionales y provinciales en relación a las normativas que refieran a EAN.

- Describir cómo se desarrollan los contenidos de EAN y cómo inciden en las trayectorias escolares de los y las estudiantes, a través de entrevistas a diferentes actores de la comunidad educativa.

- Evidenciar qué enfoques pedagógicos sustentan los abordajes de EAN en las prácticas de los y las docentes.

- Conocer la participación de profesionales idóneos en el área de nutrición y educación en tareas referidas a EAN en las escuelas y entornos escolares.

Para lograr los objetivos, se indagaron y contrastamos antecedentes de normativas, programas, guías de EAN y estudios de caso para conocer el plano micropolítico de las instituciones. Este último refiere a aquellas disputas, conflictos de intereses y relaciones intrincadas entre las políticas educativas que refieren a EAN, las instituciones, los actores y actoras que las implementan, desarrollan e interpretan (Ball, 1989). 
REVISTA DE LA ESCUELA DE CIENCIAS DE LA EDUCACIÓN, AÑO 17, NRO. 16, VOL. 1, ENERO A JUNIO DE 2021. PÁGINAS 107-117. ISSN 2362-3349 (EN LíNEA). EDUCACIÓN ALIMENTARIA NUTRICIONAL (EAN) COMO CONTENIDO TRANSVERSAL EN PERSPECTIVA DE POLÍTICA PÚBLICA EN INSTITUCIONES DE NIVEL INICIAL Y PRIMARIO DE LA CIUDAD DE ROSARIO, PROVINCIA DE SANTA FE. VICTORIA BRUCH.NATALIA RETAMAR MALIZIA.ILEANA SILVIA SORGENTONI.

\section{Marco teórico conceptual y referencial}

\section{Normativa internacional}

El derecho a la alimentación es reconocido por los tratados internacionales de derechos humanos. Entre ellos la Declaración Universal de Derechos Humanos de la Organización de las Naciones Unidas (ONU), vigente desde 1948, así como el Art. 24 de la Convención sobre los Derechos del niño (Organización de las Naciones Unidas, 1989).

En nuestro país, todos estos derechos están reconocidos dentro de la Constitución Nacional, en el artículo 75 , inciso $22^{2}$.

\section{Normativa nacional}

A través de la Resolución 732/2016 el Ministerio de Salud de la Nación crea el Programa Nacional de Alimentación Saludable y Prevención de la Obesidad. Así se conforma la Comisión Nacional de Alimentación Saludable y Prevención de la Obesidad, integrada por diversos actores pertenecientes a organismos dependientes de dicho Ministerio, además de profesionales, universidades, entidades científicas, académicas, organismos no gubernamentales e instituciones vinculadas con la problemática de alimentación.

En el año 2019, el Ministerio de Salud y Desarrollo Social de la Nación, a través de la Secretaría de Gobierno de Salud, crea por Decreto № 996/19 el Plan Nacional de Alimentación Saludable en la Infancia y la Adolescencia para la Prevención del Sobrepeso y la Obesidad en Niños, Niñas y Adolescentes (Plan ASI). El mismo se propone detener la epidemia creciente de sobrepeso y obesidad en niños, niñas y adolescentes en el país para el año 2023 mediante una serie de iniciativas, dentro de las cuales se incluye la EAN.

En el marco del Plan Argentina Contra el Hambre, en el año 2020, se brindaron capacitaciones virtuales gratuitas para docentes sobre huertas agroecológicas en la escuela y alimentación saludable.

\section{Normativa de la provincia de Santa Fe}

En noviembre de 2012, la Legislatura de la Provincia de Santa Fe sanciona la Ley $n^{\circ} 13295$, mediante la cual crea el Programa Provincial Integral de Prevención, Control y Asistencia de los Trastornos Alimentarios, en adhesión a la Ley Nacional № 26396. Esta norma establece la incorporación de la EAN en el sistema educativo en todos los niveles; la promoción de la actividad física; el control sobre los quioscos, buffet; la fijación de criterios y acciones de capacitación y actualización de los docentes de los distintos niveles, ciclos o modalidades sobre esta problemática; entre otras.

En febrero de 2018 se promulga en la provincia de Santa Fe la Ley no 13719 con el objetivo de promover la alimentación saludable por medio de la EAN en niños, niñas y adolescentes que concurren a escuelas públicas y privadas. Esta ley aún no está reglamentada.

\section{Documentos oficiales que guían la EAN}

La Organización Mundial de la Salud (OMS) y la Organización Panamericana de la Salud (OPS) han desarrollado diversos documentos en los que recomiendan mejorar el entorno escolar con respecto a la nutrición y la actividad física, implementar un impuesto sobre las bebidas azucaradas, difundir directrices nutricionales para toda la población, regular la publicidad de alimentos y bebidas altos en grasas, azúcar y sal, implementar un etiquetado en el frente de los envases de alimentos que sea fácil de interpretar (OMS 2004; OMS, 2016).

A nivel nacional, el principal documento oficial son las Guías Alimentarias para la Población Argentina (GAPA), publicadas en 2016. Estas presentan una herramienta de educación nutricional cuyo propósito es contribuir a la prevención de ECNT y prevenir problemas asociados a una mala alimentación (MSAL, 2016).

En 2019 se publicó la Guía de Entornos Escolares Saludables elaborada por el Programa Nacional de Alimentación Saludable y Prevención de Obesidad y el Programa Nacional de Lucha contra el Sedentarismo. El objetivo de la misma es establecer un conjunto de recomendaciones que, basadas en las evidencias científicas disponibles, constituyan los estándares para guiar el proceso de sanción de leyes e implementación de programas efectivos, para transformar los entornos obesogénicos en entornos escolares saludables (Ministerio de Salud y Desarrollo Social de la Nación y Ministerio de Educación de la Nación, 2019).

En el año 2016, FAGRAN, emitió un documento recopilatorio de las conclusiones elaboradas a partir de la Reunión Nacional de Alimentación Escolar del año 2013. Entre otras cuestiones se identifica la falta de licenciados/as en nutrición en los Programas de Alimentación Escolar; y de normativa relacionada. Se presentan recomendaciones para la evaluación nutricional de alumnos/as, planificación de servicio de alimentación en comedores escolares, y los recursos, contenidos y modalidades necesarios para la

${ }^{2}$ Para ampliar información consultar: http://servicios.infoleg.gob.ar/infoleglnternet/anexos/0-4999/804/norma.htm 
aplicación de EAN en las escuelas. FAGRAN sugiere incorporar licenciados/as en nutrición para la realización de actividades de EAN en el ámbito escolar.

El Ministerio de Desarrollo Social de Santa Fe, a través de la dirección provincial de Seguridad Alimentaria, presentó la segunda edición de la "Bitácora de la Alimentación Segura y Saludable" en el año 2019. Es un material con técnicas y estrategias didácticas elaborado por un grupo de trabajadoras de los Ministerios de Desarrollo Social, Educación y Salud donde se busca compartir estrategias para pensar, reflexionar, construir saberes relacionados con la alimentación; donde el juego es protagonista, la cultura mediadora y el encuentro una manera de enlazar ideas y propuestas (Ministerio de Desarrollo Social de la Provincia de Santa Fe, 2019).

\section{EAN en Trayectorias Escolares}

Los avances del conocimiento han ido cambiando con el transcurso del tiempo, y con ello, las redefiniciones de los contenidos socialmente válidos para enseñar y construir en las instituciones sobre los trayectos escolares. Por contenido entendemos al conjunto de saberes, códigos, lenguajes, situaciones problemáticas sociales y culturales que son puestas como objetos de estudio para enseñar sufriendo una transformación del contenido erudito al contenido a ser enseñado en las instituciones (Chevallard, 1991). Los contenidos no se desarrollan como neutros o naturalizados (Taborda, 1951).

La Organización Mundial de la Salud (OMS) y la Organización de las Naciones Unidas para la Alimentación y la Agricultura (FAO) aluden que EAN no contempla sólo la difusión de información acerca de los alimentos y sus nutrientes, sino que también proporciona herramientas para saber qué hacer y cómo actuar para mejorar la nutrición, proporcionando las capacidades necesarias para que las personas puedan alimentarse y alimentar a sus familias adecuadamente, realizar compras seguras a precios accesibles, preparar alimentos y comidas saludables que sean de su agrado, educar a sus hijos/as sobre alimentación saludable. Por este motivo EAN es concebida como "la combinación de experiencias de aprendizaje diseñadas para facilitar la adopción voluntaria de conductas alimentarias y otras conductas relacionadas con la nutrición, que conduzcan a la salud y al bienestar" (FAO y OMS, 1992). A su vez, la Asociación Americana de Dietética (ADA), asume el mismo significado (Shafer y col., 1996). EAN necesariamente debe pensarse como política pública, aquella que atañe un proceso complejo y plural con múltiples actores públicos y privados cruzados y envueltos en relaciones de cooperación, competencia y/o conflicto (Bentancur y Mancebo, 2012). De estos procesos no ha estado desprovista la educación. En el año 2004, el Consejo Federal de Cultura y Educación (CFCyE), señala su preocupación por la fragmentación y desigualdad en todo el territorio del país por la herencia de políticas neoliberales implementadas entre los años 1989 y 2001. En el año 1997, se concretan los diseños curriculares de los niveles que nos competen en nuestra investigación. En el Nivel Inicial, se atribuye un espacio para pensar la alimentación como complemento más del desarrollo de autonomía de los/as niños/as en donde el docente debe acompañar ese proceso como una actividad cotidiana, tal como el baño y la vestimenta. En lo que respecta al diseño de Nivel Primario (Primer Ciclo), hay enunciaciones de la alimentación como un concepto que acompaña la descripción de algún elemento, tiempo histórico o registro sobre los procesos de la historia, la tecnología, la matemática, etc ${ }^{3}$. Por todos estos diseños que generaron grandes fragmentaciones al sistema educativo del país, existía la necesidad de implementar políticas para reconstruir y recuperar la centralidad del Estado en materia de enseñanza y que promuevan aprendizajes en el sentido de construcción de ciudadanía, justicia social, igualdad y solidaridad. A tal fin, se emite por unanimidad la Resolución Ministerial $N^{\circ}$ 214/2004, de la cual participaron representantes políticos, técnicos, supervisores y docentes de las provincias argentinas, de la Ciudad de Buenos Aires y de los equipos técnicos del Ministerio de Educación, Ciencia y Tecnología de la Nación. En este documento se acuerda la identificación de un Núcleo de Aprendizajes Prioritarios (NAP) 4 . Los NAP están divididos por ciclos de espacio entre compañeros/as como un primer encuentro con la organización formal escolar del sistema educativo. Los NAP de Primer Ciclo están organizados por campos de conocimiento y por año. Su formulación incluye los saberes que se propone promover para el ciclo de enseñanza en esos campos. Estos diseños actúan a modo de guías para que cada provincia e institución pueda desarrollar la metodología que crea conveniente. En lo que respecta a la provincia de Santa Fe, de acuerdo a la Ley Nacional de Educación (LEN 26.206), en el 2016 se gestan los Núcleos Interdisciplinarios de Contenidos (NIC) y se concretan en 2017. Basados desde una perspectiva socio inclusiva, al igual que los $\mathrm{NAP}^{5}$, pregonando una enseñanza problematizadora que se enriquece con la construcción de trabajo interdisciplinario, sobre una problemática y acontecimiento social. Estos

${ }^{3}$ Consulta a base documental de los primeros diseños de la provincia de Santa Fe en el año 1997. La Comisión de Diseño Curricular se crea por Resolución Ministerial Provincial ํํ45/96, dependiente de la Dirección Provincial de Educación Superior, Perfeccionamiento Docente, Programación y Desarrollo Curricular. Comienza a trabajar en mayo de 1996, estableciendo las pautas y las estrategias para la conformación del equipo cuyo objetivo es la elaboración de los Diseños Curriculares Jurisdiccionales de Nivel Inicial, EGB (primer ciclo, segundo ciclo, tercer ciclo) y Polimodal. http://www.bnm.me.gov.ar/giga1/documentos/EL000363.pdf/

http://www.bnm.me.gov.ar/giga1/documentos/EL000365.pdf

${ }^{4}$ Consulta documentos referidos a Nivel Inicial y Nivel Primario Primer Ciclo http://repositorio.educacion.gov.ar:8080/dspace/bitstream/handle/123456789/94247/nap-nivel_inicial.pdf?sequence=1 ${ }^{5} \mathrm{http}: / /$ www.bnm.me.gov.ar/giga1/documentos/EL000977.pdf 
documentos tratan de que cada docente e institución pueda desarrollar los contenidos bajo su perspectiva, "cultura e identidad institucional" (Fernández, 1995). Son propulsores del "constructivismo" porque retoman los conocimientos y experiencias previas de las personas en situación de aprendizaje enfatizando al conocimiento como producto de una interacción sociocultural en donde las personas construyen, reconstruyen, debaten, contrastan, elaboran, crean (Ausubel, 1976; 2002). Se basan en la "metodología de la problematización de problemas" (Davini, 2008) y abonan procesos pedagógicos desde la dialogicidad, la cooperación, desterrando prácticas aisladas, fragmentadas, intentando superar planes de estudios mosaicos con asignaturas separadas sin intervención y articulación entre ellas.

\section{Perspectivas pedagógicas en salud: una necesaria revisión crítica y transformadora}

La Educación para la Salud (EpS) y la Educación Alimentaria Nutricional (EAN) han transitado una construcción diferente en diversos tiempos y ello ha diseñado sus concreciones sobre la sociedad. Varios autores y autoras, acercan un estado del arte sobre estas conceptualizaciones, mencionando el europeo y norteamericano como dominante $u$ hoja de ruta donde ha primado ese modelo mundialmente influenciado por el paso por diferentes enfoques pedagógicos en salud. Primeramente, tenemos las iniciales en esos contextos que refieren al siglo XIX en relación al modelo higienista o el tradicional que se define con una concepción positivista de la ciencia y una concepción biomédica de la salud responsabilizando individualmente al paciente de las conductas como efecto causa, pensando como factor individual, conductual y biológico el determinante central de su enfermedad (Peñaranda y col., 2017). Según este enfoque, el profesional de la salud se presenta con mirada biologicista unicausal y el rol del paciente es el de un objeto pasivo a diagnosticar, corregir y analizar6. Hay un análisis simplista y reduccionista de la realidad, del contexto y la subjetividad. En otro posicionamiento, hay corrientes (herederas de la educación popular en el caso de las corrientes críticas latinoamericanas) que se sustentan en una perspectiva crítica de ciencia y salud, y conciben a esta última como producto de las condiciones sociales, culturales, económicas y políticas, entendiendo tanto al profesional de la salud, como al paciente, sujetos en contexto implicados como actores de transformaciones de las problemáticas de salud de manera conjunta. En este sentido, Alzate Yepes (2013), a través de un estudio cualitativo documental analiza algunas experiencias en salud en correlación con los enfoques, tendencias o perspectivas pedagógicas en las que se sustentan. Expone que la gran mayoría de las experiencias no cuentan con referentes pedagógicos explícitos y, de aquellas que los tienen, pudo verificar una gran contradicción en la cohesión de las ideas y las estrategias abordadas; incoherencias al desarrollar estrategias educativas que corresponden a modelos o teorías diferentes a las propuestas. Releva que se enfocan más en los contenidos que en el método de enseñanza; evalúan la adquisición de conocimientos de forma cuantitativa; y la educación se centra en enseñar el qué (causas de enfermedades) y no en el cómo prevenir o resolver problemas de salud. Pone el acento en una mirada hacia un sujeto integral, abordando los problemas de salud de manera multidimensional, multirreferencial y poliocular. Busca la formación de profesionales críticos que asuman la multicausalidad, multirespuesta, multisectorialidad, multidisciplinariedad y la participación social, con el respeto de la autonomía del sujeto y la responsabilidad colectiva en salud.

\section{Diseño metodológico}

Este estudio de caso en profundidad de un caso en particular (Fica, 2005) se enmarca dentro de los enfoques cualitativos ${ }^{7}$ de tipo descriptivo, exploratorio y de corte transversal. Los datos obtenidos fueron recolectados por selección y análisis documental en relación a EAN respecto de su incidencia en Nivel Inicial y Primario (Primer ciclo), con mayor énfasis en normativas y documentos que rigen en todo el país y la provincia desde 2016. Se realizaron nueve entrevistas etnográficas ${ }^{8}:$ tres docentes pertenecientes a cada escuela, tres directoras de los niveles, dos referentes del Colegio de Graduados en Nutrición 1ํㅜ Circunscripción de Santa Fe y el equipo técnico del servicio de alimentación de la Dirección Provincial del Programa Alimentario del Ministerio de Desarrollo Social de la Provincia de Santa Fe.

\section{Resultados de entrevistas etnográficas}

\section{Actores de la comunidad educativa}

Las tres instituciones adhieren no estar capacitadas para abordar los contenidos de manera transversal ni en forma de política pública porque no hay, según expresan, "una necesaria revisión de los hábitos alimentarios de los/as niñas/niños en relación con lo que traen de casa y lo que tienen". Indican que tampoco hay un abordaje entre el Ministerio de Salud, el de Desarrollo Social o el de Educación. Más allá

\footnotetext{
${ }^{6}$ Se puede identificar nociones de higiene bajo un paradigma tradicional que va del siglo XV AL XVII, aquella corriente pedagógica magistrocéntrica verbalista, memorística, metódica, autoritaria, enciclopédica, donde educandos tienen un rol de pasividad para recibir instrucción como enseñanza-aprendizaje. Los inspectores técnicos y de Higiene del Consejo Nacional de Educación quedan comprendidos en el artículo 56 de la ley 1420 del 8 de julio de 1884. A su vez, se puede identificar más precisiones referidos a la misma en otros como el №, 13, 42, 70 y 75 . http://www.bnm.me.gov.ar/giga1/documentos/EL002646.pdf

${ }^{7}$ El método de investigación cualitativo es una producción constructiva-interpretativa, donde el sujeto es protagonista en la construcción y reconstrucción del conocimiento científico, que permite incorporar lo subjetivo. Estos métodos no solo describen la realidad, sino que la construyen, e incluyen al investigador como parte de él (Fica, 2002).

${ }^{8}$ Ver (Guber: 2001). La etnografía. Método, campo y reflexividad. Grupo editorial NORMA. Buenos Aires. En el capítulo N4: "La Entrevista Etnográfica el arte de la no directividad".
} 
de la consulta de normativas, programas, organismos, libros y diseños curriculares, las personas entrevistadas adscriben que no tienen un trabajo en equipo interdisciplinar para abordar EAN en la escuela adentrándose en las costumbres, gustos, necesidades y particularidades de cada familia en cada contexto ${ }^{9}$. Otra de las debilidades que encuentran es no saber en qué momento del año poder abordar el contenido como proyecto, unidad o secuencia didáctica. Pocos actores de la comunidad abordan y conocen libros para pensar la misma desde la complejidad y el territorio. En ningún caso se evidencia capacitación referida a las diferentes opciones y líneas de abordaje que proponen algunos cuadernos saludables, guías o leyes nacionales sobre EAN. De las entrevistas a los/as docentes, con mayor énfasis en el Nivel Inicial, denotan los cuidados de higiene, reconocimiento de alimentos, valoración sobre una correcta hidratación, identificación y diferenciación de legumbres y frutas. Algunos/as docentes desarrollan estrategias de métodos inductivos y deductivos para enseñar, abordando contenidos de organismos como la OMS, documentos curriculares como los NAP, o materiales como las GAPA para lograr una aprehensión de conocimientos y comportamientos alimentarios nutricionales equitativos y saludables. Otros/as utilizan el método del diálogo reflexivo para hacer pensar en la valoración personal sobre algún alimento o elaboración de alimentos en particular, otros utilizan una problemática actual del ecosistema, por ejemplo, para abordar como contenido situado en EAN. Cuando se les pregunta a docentes del Nivel Primario por las estrategias metodológicas sobre la implementación y abordaje de EAN de sus clases, ellas responden que suelen ser de tipo expositivas, comparativas, que apelan a la dialogicidad reflexiva en muchos casos; pero para establecer un cambio conceptual, una metodología que fortalezca el razonamiento crítico ante el conocimiento y la mejora de la calidad de vida, coinciden en que falta un abordaje en comunidad, en equipo interdisciplinar también. En el Nivel Primario, en base a las respuestas de los/as directivos/as, se utilizan también prácticas para abordar EAN de manera expositiva y, a veces, problematizadora con historias de las distintas culturas de nuestro país y sus costumbres. En el nivel inicial, EAN se aborda en espacios de huerta pensando en el cuidado de las aromáticas, reciclado, confección de manualidades y allí mismo plantean las propiedades y características de los alimentos, inclusive del agua, mediante la recolección de la misma en un espacio en la huerta. La apropiación de los contenidos sobre EAN propicia lo kinestésico y la estimulación motriz en los/as niños/as favoreciendo su adaptación con los pares. Las prácticas relatadas, mayormente en Nivel Primario, fueron exposiciones dialogadas con filminas y, en algunos casos, con muestras de plástico sobre las mesas en donde cada estudiante podía tocar y reconocer características de algunos alimentos por sobre otros. En los dos niveles, el contexto de vulnerabilidad, genera que algunos/as niños/as no participen de la clase aún pese a la disposición en ronda que habilita corporalmente a la exploración del espacio y de los pares por la dificultad en el reconocimiento de algunos procesos sobre los alimentos. Por otro lado, en algunos hogares prevalece una alimentación de carácter monótona; es decir, no cumplen con las comidas en tiempo, variedad y calidad como requerirían sus necesidades. Los/as docentes de este nivel educativo tienen grandes preocupaciones por esto, ya que los niños en varias ocasiones, se duermen y no están atentos debido a la mala calidad e insuficiente cantidad de alimentos consumidos. También indican que los estudiantes, esperan ir al comedor escolar para acceder a una ración de comida que, generalmente, se establece en el horario de las 11:00 AM. Ello irrumpe en la labor docente y en el desempeño de los estudiantes. En el Nivel Inicial, el directivo percibe menos diferencias que en niveles posteriores porque, anteriormente a los protocolos por la pandemia por COVID-19, en los jardines se compartían los alimentos, favoreciendo un ambiente de equidad e igualdad. No se traían alimentos que otros padres, madres o tutores, no pudieran comprar. Hay una atención permanente y sensible por parte de la comunidad educativa para favorecer procesos democráticos e igualitarios en los/as alumnos/as. Hay menos segmentación y desigualdad. En este nivel, se identifica que los/as niños/as aprenden contenidos sobre el cepillado dental, lavado de manos y otros hábitos de higiene, lo cual genera una contradicción en muchas situaciones en el contexto donde viven porque, en sus casas, algunos/as no tienen condiciones edilicias habitables ni cuentan con los recursos necesarios para sanitizarse. En esto los/as docentes reparan y, muchas veces, compran los materiales. Todos/as los/as actores asumen que los NAP son completos para explicar la alimentación de los seres vivos, y son más consultados en espacios de ciencias naturales. En una escuela en particular, al tener una docente de primaria licenciada en nutrición, elaboraron dos proyectos conjuntos para enseñar EAN con diferentes colegas de educación física, artes y música. A través de relatos de los/as niños/as identifican y valoran la importancia de las comidas en familia y sus gustos

\footnotetext{
${ }^{9}$ Requerimos lectura EAN para las instituciones porque los mismos intentan establecer trabajo interdisciplinar, didáctico colectivo y colaborativo entre familias y comunidades empezando desde los espacios áulicos de las escuelas para transformar las prácticas la sobre lacación alimentaria. http://www.bnm.me.gov.ar/giga1/documento/EL0096;002085;00997y 0098
} 
particulares. Propulsaron que los/as niños/as, en diferentes disciplinas, pudieran pensar los alimentos como construcción socio cultural en contexto e interpelando los modos de consumo.

\section{Responsables del Servicio de Alimentación del Ministerio de Desarrollo Social de Rosario}

El servicio está conformado por: ocho nutricionistas, una docente, una terapista ocupacional, una psicopedagoga y una psicóloga en toda la provincia de Santa Fe. Realizan actividades de formación y acompañamiento a las instituciones dependientes del Ministerio de Desarrollo Social (Centros de acción Familiar, Residencia de niñas, niños y adolescentes y Residencias de adultos mayores), y a las organizaciones e instituciones vinculadas o interesadas en la temática, a lo largo del territorio provincial. También supervisan los servicios de alimentación, desarrollan talleres de EAN para el personal de dichas instituciones y para las personas que asisten a ellas, capacitaciones en manipulación de alimentos, entre otras. Además, articulan acciones con el Ministerio de Seguridad (capacitación y asesoramiento en alimentación saludable y en la implementación de la Tarjeta Alimentaria); así como con el Ministerio de Salud (jornadas, actividades y eventos).

\section{Colegio de Graduados en Nutrición 1ra. Circunscripción de la Provincia de Santa Fe}

Se indagó sobre la participación del Colegio en el proyecto de Ley №13.719. Sus representantes refirieron que al notificarse del mismo se contactaron con los funcionarios que lo impulsaron. "Observamos que el proyecto tenía varios errores y desde el Colegio formamos una comisión para trabajarlo". En conjunto con estos y con nutricionistas del área se elaboraron observaciones para que sean consideradas antes de aprobar la ley. "La ley fue aprobada, si mal no recuerdo, un 28/12/2017 sin las modificaciones sugeridas o habiendo realizado muy poquitas de ellas. Nos pusimos en contacto con funcionarios para manifestar nuestra disconformidad, quienes se disculparon y propusieron convocarnos para hacer la reglamentación. Desde ese momento no hemos recibido invitación para ello y tengo entendido que esa ley sigue sin reglamentarse".

En diálogo con otra autoridad de la misma institución, comentó que en el 2020 ambos Colegios de Graduados en Nutrición (1aㅡ y $2^{\circ}$ Circunscripción) han sido convocados por otro sector y han colaborado en un proyecto de modificación de varios puntos de esta ley, el cual se encuentra hoy en tratamiento en comisiones en la legislatura provincial.

\section{Discusión}

EAN es un área de estudio en sí misma y debería pensarse en coherencia con el entorno escolar (el aula no es el único espacio en el que se educa). El comedor, el quiosco y la cantina del colegio son espacios donde necesariamente debe trabajarse EAN y en las instituciones estudiadas esto no se logra implementar. Es necesario trascender la frontera de lo meramente institucional y considerar las múltiples circunstancias que inciden en cómo se alimentan los/as estudiantes dentro y fuera de la escuela porque impacta en sus desempeños y actividades de la vida diaria. Los factores que afectan sus decisiones alimentarias, se vinculan con la disponibilidad y acceso a los alimentos sanos, la publicidad de comestibles ultraprocesados, las circunstancias del consumo, los significados sociales de la comida, el compartir o no la mesa familiar, la situación socioeconómica, política, social y estructural a nivel individual y colectivo. Por tanto, las políticas públicas vinculadas a la producción de alimentos, su distribución y comercialización inciden en el consumo. El problema de la malnutrición requiere no sólo tratamiento, sino también prevención. Hace tan solo 15 años que el Estado inició acciones para evaluar a la población en relación al estado nutricional con la primera ENNyS en 2005, la segunda ENNyS en 2019 y una serie de Encuestas Nacionales de Factores de Riesgo de ECNT que se iniciaron también en 2005. Éstas han sido el punto de partida para el desarrollo de documentos que guían la EAN a nivel poblacional oficiando como herramienta sanitaria para la promoción de la salud y la prevención de ECNT relacionadas con la alimentación en nuestro país. No obstante, la malnutrición sigue creciendo y difícilmente se detenga si se aplican medidas aisladas. Como se ha visto en el marco normativo, hay interés por parte de organismos internacionales, nacionales y provinciales respecto a este tema, pero hay una evidente dificultad a la hora de concretar acciones que hagan frente a los problemas sanitarios y educativos que aquí se tratan. En consonancia con las conclusiones de la Reunión Nacional de Alimentación Escolar del año 2013, se evidencia que las falencias que entonces se denunciaron persisten al día de hoy en los casos estudiados. En este sentido, es necesario generar espacios donde se desarrolle EAN inter y transdisciplinariamente al tiempo que se mejoren los entornos escolares. Es urgente intervenir en la publicidad de comestibles ultraprocesados, en la comercialización de alimentos en las cantinas escolares y destinar suficientes recursos económicos a la compra de alimentos sanos para mejorar la calidad de los menús de los comedores escolares y copas de leche. Con la información que ofrecen los casos, entendemos que el alimento que es ofrecido en el comedor no complementa la alimentación del hogar, sino que muchas veces es lo único a lo que los/as estudiantes tienen acceso en el día, con lo cual es más importante aún atender su calidad ${ }^{10}$.

\footnotetext{
${ }^{10}$ Tesina de grado presentada por estudiantes de Lic. en Nutrición de la Universidad de Concepción del Uruguay, sede Rosario, realizaron una valoración nutricional de los menús de dos comedores de dos escuelas públicas de la ciudad de Rosario. Evidenció que los menús no cumplían con las recomendaciones oficiales de FAGRAN, por bajo presupuesto
} 
En los NAP, NIC y los Cuadernos Saludables, se expone a la alimentación como inherente a todo ser vivo y al ser humano en particular, influenciado por el contexto histórico, político, cultural y social. Colocan a la EAN en un espacio importante en la educación porque revisan desde una postura crítica y transformadora cómo ha ido cambiando la misma a lo largo del tiempo. Proponen que la alimentación sea abordada desde diferentes disciplinas como ser: histórica, política, biológica, química, económica, cultural, psicológica y social. En el capítulo que hace referencia a la alimentación, los NIC, interpelan los modos del consumismo mercantilista propios de la globalización en relación a la compra de comida chatarra y sus consecuencias en la salud; como también el lugar de la mujer, en relación a las prácticas económicas, estereotipos de belleza, patrones culturales y subjetivantes. Sin embargo, se pudo analizar que no existe en las normativas oficiales nacionales o provinciales desde el año 2016, abordaje transversal sobre EAN en clave de política pública que permita un aprendizaje integral desde Nivel Inicial hacia estudios superiores; tampoco en articulación entre un nivel y otro. A la EAN, se la concibe como un contenido específico más para abordar. Se evidencian prácticas aisladas sin poder pensar en un desarrollo donde los/as niños/as aprendan que EAN es imprescindible para una mejor calidad de vida, fortaleciendo los procesos de enseñanza y aprendizaje.

\section{Reflexiones y propuestas}

EAN no forma parte de la currícula obligatoria como campo transversal de conocimiento abordado desde una forma inter y transdisciplinaria con otras instituciones asociadas para el fortalecimiento de dicho espacio. Hay valiosas intenciones de la comunidad educativa por concretar EAN en ambos niveles, pero se aborda con poco margen de tiempo en el año quedando reducido al plano biológico, y con gran dificultad al acceso de conocimiento sobre bases críticas por falta de capacitaciones y trabajo mancomunado entre ministerios. Los enfoques pedagógicos en que se sustentan las clases mayormente son tradicionales: expositivos, inductivos y verbalistas con poco margen para el enriquecimiento colectivo. Por esto se considera necesario implementar:

- Establecer EAN de manera transversal en todas las instituciones educativas públicas y privadas, con abordaje inter y transdisciplinario.

- Creación de gabinetes con profesionales de la salud y de la educación en cada institución.

- Implementación de enfoques y abordajes sobre EAN críticos y emancipadores, comprendiendo el respeto a la construcción de saberes y la cultura alimentaria de las comunidades, la valoración de la economía doméstica, el cuidado del ambiente ${ }^{11}$, el respeto a la diversidad cultural, con enfoque y perspectiva de género en clave de política pública ${ }^{12}$.

- Atención al presupuesto que reciben los servicios de alimentación escolar.

- Articulación entre los distintos Ministerios que permitan concretar cambios sostenibles a largo plazo y que promuevan colectivamente una mejora en la calidad de vida de los/as niños/as.

Aquí no se esboza una conclusión porque se propiciaría el cierre de la investigación. Al contrario, las autoras sostienen que queda mucho por recorrer y que EAN, como política pública, es ir más allá de lo prescripto o el desarrollo de un aula taller. Es trascender para establecer el ejercicio del derecho humano a la alimentación, a la salud y a la educación. El Estado debe garantizar EAN en todos sus niveles, específicamente en la escuela, como parte de la conformación de entornos escolares más propicios para el proceso de enseñanza y aprendizaje de niños/as. Las autoras definen EAN desde una perspectiva crítica y transformadora como un proceso dialógico horizontal dinámico de intercambio y construcción de saberes en contexto. En ella se desarrollan actividades para favorecer prácticas, comportamientos y hábitos alimentarios beneficiosos para la salud transformando aquellos que no lo son, con el fin de promover un buen estado nutricional en la infancia y adultez. Es imprescindible seguir trabajando en la construcción de una EAN que esté presente en las trayectorias escolares reales porque es el camino para que la comunidad pueda bregar por su derecho a una alimentación sana. Los/as niños/as son el futuro de nuestra población; es por esto que se propone generar un espacio de análisis, reflexión y toma de conciencia alrededor de la articulación entre salud y educación.

destinado, concluyendo que la falta de participación de personal idóneo en la materia podría contribuir con ese problema (Márquez y Teves: 2020).

${ }^{11}$ Se sugiere leer los aportes de Edgar Morin (1921) pensador y escritor francés más influyente del Siglo XX.

El libro "Los siete Saberes o Principios básicos para la Educación del futuro" (2001).

${ }^{12}$ Podemos percibir algunos supuestos a los que arribamos, dentro del programa "Alimentar Saberes" recientemente lanzado por el Ministerio de Desarrollo Social Nacional en octubre del 2020, en el marco del Plan Argentina contra el Hambre. https://www.argentina.gob.ar/noticias/lanzan-programa-nacional-de-educacion-alimentaria. 
REVISTA DE LA ESCUELA DE CIENCIAS DE LA EDUCACIÓN, AÑO 17, NRO. 16, VOL. 1, ENERO A JUNIO DE 2021. PÁGINAS 107-117. ISSN 2362-3349 (EN LíNEA). EDUCACIÓN ALIMENTARIA NUTRICIONAL (EAN) COMO CONTENIDO TRANSVERSAL EN PERSPECTIVA DE POLITICA PÚBLICA EN INSTITUCIONES DE NIVEL INICIAL Y PRIMARIO DE LA CIUDAD DE ROSARIO, PROVINCIA DE SANTA FE. VICTORIA BRUCH.NATALIA RETAMAR MALIZIA.ILEANA SILVIA SORGENTONI.

\section{Referencias bibliográficas:}

Aguirre, P. (2010). Ricos flacos y gordos pobres. La alimentación en crisis. Buenos Aires: Capital Intelectual.

Alzate Yepes, T. (2013). Desde la educación para la salud: Hacia la pedagogía de la educación alimentaria y nutricional. Perspectivas En Nutrición Humana, (16), pp. 21-40. Disponible en: https://revistas.udea.edu.co/index.php/nutricion/article/view/17866.

Ausubel, D. P. (1976). Psicología educativa. Un punto de vista cognoscitivo. México: Trillas

Ausubel, D. P. (2002). Adquisición y retención del conocimiento. Una perspectiva cognitiva. Barcelona: Paidós.

Ball, S. J. y Míguez, N. (1989). La micropolítica de la escuela: hacia una teoría de la organización escolar. Barcelona: Paidós.

Bentancur, N. y Mancebo, M.E. (2012). Políticas educativas en tiempos de cambio. Actores, e instituciones en Uruguay y la región. En: Revista Uruguaya de Ciencia Política, 21(1), 1-6. Disponible en: http://rucp.cienciassociales.edu.uy/index.php/rucp/article/view/5

Chevallard, I. (1991). La transposición didáctica. Del saber sabio al saber enseñado. Buenos Aires: Aique.

Davini, M. C. (2008). Métodos de enseñanza. Didáctica general para maestros y profesores. Buenos Aires: Santillana.

Federación Argentina de Graduados en Nutrición (FAGRAN) (2013). Conclusiones de la Reunión Nacional de Alimentación Escolar. Disponible en: http://www.codajic.org/sites/www.codajic.org/files/Conclusiones\%20de\%20la\%20Reuni\%C3\%B3n\%20Nacion al\%20de\%20Alimentaci\%C3\%B3n\%20Escolar\%202013\%20(1).pdf

Fernández, L. (1995). Instituciones educativas: dinámicas institucionales en situaciones críticas. Buenos Aires: Paidós.

Fica, R. (2005). Reflexión epistemológica: Introducción a la Investigación Cualitativa. El estudio de casos. Ficha Pedagógica NAE: Rosario

Márquez D. y Teves N. (2020). Valoración nutricional de menús elaborados en dos comedores escolares de zona norte de la ciudad de Rosario [tesina de grado]. Universidad de concepción del Uruguay, Centro Regional Rosario: Rosario.

Organización Mundial de la Salud (2004). Estrategia Mundial sobre régimen alimentario, actividad física y salud. Disponible $\quad$ en: https://extranet.who.int/iris/restricted/bitstream/handle/10665/43037/924359222X_spa.pdf;jsessionid=E80BAE F02D39D468C11D63FD1BF951A0?sequence $=1$

Organización Mundial de la Salud (2016). Informe de la Comisión para acabar con la obesidad infantil. Disponible en: https://apps.who.int/iris/bitstream/handle/10665/206450/9789243510064_spa.pdf?sequence=1

Organización Mundial de la Salud y Organización de las Naciones Unidas para la Alimentación y la Agricultura, Unidad de Nutrición (1992). Elementos principales de estrategias nutricionales. № ICN/92/INF/7.Ginebra. Disponible en: https://apps.who.int/iris/handle/10665/61052

Organización Panamericana de la Salud y Organización Mundial de la Salud (2014). Plan de acción para la prevención de la obesidad en la niñez y la adolescencia. https://www.paho.org/hq/dmdocuments/2015/Obesity-Plan-OfAction-Child-Spa-2015.pdf

Organización de las Naciones Unidas (ONU) (1989). Oficina del Alto Comisionado en Derechos Humanos. Convención sobre los Derechos del Niño. Disponible https://www.ohchr.org/sp/professionalinterest/pages/crc.aspx\#: :text=Art\%C3\%ADculo\%2024\&text=Los\%20E stados\%20Partes\%20reconocen\%20el,la\%20rehabilitaci\%C3\%B3n\%20de\%20la\%20salud

Peñaranda F.; López J. M. y Molina D.P. (2017). La educación para la salud en la salud pública: un análisis pedagógico. En: Hacia la promoción de la Salud. 22(1): 123-133. DOI: 10.17151/hpsal.2017.22.1.10

Shafer L.; Gillespie A.; Wilkins J.L. y Borra S.T. (1996). Posición de la Asociación Americana de Dietética: Educación Nutricional para el Público. Elsevier Inc. En: Revista de la Academia de Nutrición y Dietética, 96(11), 11831187. Disponible en: https://doi.org/10.1016/S0002-8223(96)00305-7

Taborda, S. (1951). Investigaciones Pedagógicas, Volumen I y II. Córdoba: Ediciones Ateneo Filosófico de Córdoba.

Torresani, ME (2010). Cuidado Nutricional Pediátrico. Buenos Aires: Eudeba.

Zapata ME., Rovirosa A., y Carmuega E. (2016). La mesa Argentina en las últimas dos décadas: cambios en el patrón de consumo de alimentos y nutrientes 1996-2013. Ciudad Autónoma de Buenos Aires: Centro de Estudios sobre Nutrición Infantil - CESNI.

\section{Normativas y documentos:}

Ley Nacional N²6061. Ley de protección integral de los derechos de las niñas, niños, y adolescentes. 21 de octubre de 2005. Publicada en el B.O. el 26 de octubre de 2005

Ley Nacional N²6206. Ley de Educación Nacional. 27 de diciembre de 2006. Publicada en el B.O. el 28 de diciembre de 2006.

Ley Nacional $\mathrm{N}^{\circ} 26396$. Por la cual se declara de interés nacional la prevención y control de los trastornos alimentarios. 13 de agosto de 2008. Publicada en el B.O. el 3 de septiembre de 2008.

Ley Provincial №13295. Por la cual la Provincia de Santa Fe adhiere a la Ley Nacional 26396 de Prevención y Control de Trastornos Alimentarios. Se crea el Programa Provincial Integral de Prevención, Control y Asistencia de los Trastornos Alimentarios. 22 de noviembre de 2012. Publicada en B.O. el 3 de diciembre de 2012.

Ley Provincial №13719. Por la cual promueve la alimentación saludable de la población de la provincia de Santa Fe, por medio de la educación alimentaria y nutricional prioritariamente de los niños, niñas y adolescentes que concurren a establecimientos escolares públicos y de enseñanza privada. 30 de noviembre de 2017. Publicada en el B.O. el 10 de febrero de 2018.

Ministerio de Desarrollo Social de la Provincia de Santa Fe (2019). Bitácora de la Alimentación Saludable: Técnicas y http://www.santafe.gob.ar/archivos/desarrollo_social/BitacoradelaAlimentacionSaludableySegura.pdf

Ministerio de Educación, Ciencia y Tecnología de la Nación (2004). Núcleos de Aprendizajes Prioritarios. Disponible en: http://www.bnm.me.gov.ar/giga1/documentos/EL000977.pdf

Ministerio de Educación de la Provincia de Santa Fe (2016). Núcleos Interdisciplinarios de Contenidos. La educación en acontecimientos. Disponible https://plataformaeducativa.santafe.edu.ar/moodle/secciones/programa_destacado.php?id=98

Ministerio de Salud de la Nación (2005). Encuesta Nacional de Nutrición y Salud. Guía para la elaboración de análisis e informes. http://www.unsa.edu.ar/bibsalud/descargas/Guia_para_la_elaboracion_de_analisis_e_informes.pdf 
Ministerio de Salud de la Nación (2006). Encuesta Nacional de Factores de Riesgo 2005. Informe de Resultados. Versión Breve. Disponible en: http://www.msal.gob.ar/images/stories/bes/graficos/0000000553cnt-2014-10_encuestanacional-factores-riesgo-2005_informe-breve-final.pdf

Ministerio de Salud de la Nación (2011). Segunda Encuesta Nacional de Factores de Riesgo para Enfermedades No Transmisibles 2010. Disponible en: http://www.msal.gob.ar/images/stories/bes/graficos/0000000570cnt-201410 encuesta-nacional-factores-riesgo-2011 informe-final.pdf

Ministerio de Salud de la Nación (2015). Tercera Encuesta Nacional de Factores de Riesgo para Enfermedades No Transmisibles 2013. Instituto Nacional de Estadística y Censos (INDEC). Disponible en: http://www.msal.gob.ar/images/stories/bes/graficos/0000000544cnt-3ra-encuesta-nacional-factoresriesgo_2013_informe-completo.pdf

Ministerio de Salud de la Nación (2016). Guías Alimentarias para la Población Argentina. Documento Técnico Metodológico. Disponible en: http://www.msal.gob.ar/images/stories/bes/graficos/0000001007cnt-201706_guia-alimentaria-poblacion-argentina.pdf

Ministerio de Salud y Desarrollo Social de la Nación. Secretaría de Gobierno de Salud. (2018). Resumen Ejecutivo Total Nacional de la Encuesta Mundial de Salud Escolar 2018. Disponible en: http://www.msal.gob.ar/images/stories/bes/graficos/0000001787cnt-emse-2018.pdf

Ministerio de Salud y Desarrollo social de la Nación. Secretaría de Gobierno de Salud (2019a). Cuarta Encuesta Nacional de Factores de Riesgo. Informe Definitivo. Disponible en: http://www.msal.gob.ar/images/stories/bes/graficos/0000001622cnt-2019-10_4ta-encuesta-nacional-factoresriesgo.pdf

Ministerio de Salud y Desarrollo Social de la Nación. Secretaría de Gobierno de Salud. (2019b). Segunda Encuesta Nacional de Nutrición y Salud. Indicadores Priorizados. Disponible en: http://www.msal.gob.ar/images/stories/bes/graficos/0000001602cnt-2019-10_encuesta-nacional-de-nutriciony-salud.pdf

Ministerio de Salud y Desarrollo Social de la Nación. Secretaría de Gobierno de Salud. (2019c). Valoración antropométrica al inicio y al finalizar el ciclo de educación primaria en Argentina. Disponible en: http://www.msal.gob.ar/images/stories/bes/graficos/0000001405cnt-Valoracin-antropomtrica-inicio-y-alfinalizar-ciclo-educacin-primaria-Argentina-07-03-2019.pdf

Ministerio de Salud y Desarrollo Social de la Nación y Ministerio de Educación de la Nación (2019). Guía de Entornos Escolares Saludables. Recomendaciones para la implementación de políticas de prevención de sobrepeso y obesidad en niños, niñas y adolescentes en instituciones educativas. Disponible en: http://www.msal.gob.ar/images/stories/bes/graficos/0000001439cnt-2019-06_entornos-escolaressaludables.pdf

Ministerio de Salud de la Nación (2020). Secretaría de Acceso a la Salud. Subsecretaría de Estrategias Sanitarias. Dirección Nacional de Abordaje Integral de Enfermedades No Transmisibles. Dirección Nacional de Abordaje por Curso de Vida. Dirección de Salud Perinatal y Niñez. Consejo Nacional de Coordinación de Políticas Sociales. Disponible en: https://www.argentina.gob.ar/noticias/capacitacion-para-docentes-sobrealimentacion-y-derecho-la-salud https://cursos.infd.edu.ar/cursos/450

Resolución Ministerial 732 de 2016 [Ministerio de Salud de la Nación]. Por la cual se crea el Programa Nacional de Alimentación Saludable y Prevención de la Obesidad. 6 de junio de 2016.

Resolución Ministerial 996 de 2019 [Ministerio de Salud y Desarrollo Social de la Nación]. Por la cual se crea el Plan Nacional de Alimentación Saludable en la Infancia y Adolescencia para la Prevención del Sobrepeso y Obesidad en Niños, Niñas y Adolescentes (PLAN ASí). 19 de junio de 2019. 